\title{
THE SPAN AND SEMISPAN OF SOME SIMPLE CLOSED CURVES
}

\author{
KATARZYNA TKACZYŃSKA \\ (Communicated by James E. West)
}

\begin{abstract}
The spans of simple closed curves that are boundaries of convex regions are determined. The following question of Lelek is answered affirmatively for these curves: Are the span and the semispan of a simple closed curve equal? The same answer is obtained in the case of nonconvex quadrilaterals.
\end{abstract}

\section{INTRODUCTION}

The concept of the span of a metric space $X$ was introduced by A. Lelek [1]. In [2], he defined three other versions of span called surjective span, semispan, and surjective semispan. In this paper we concern ourselves with the span of the boundaries of convex regions in the plane. We also deal with the simple case of a nonconvex polygon.

We begin by recalling the definitions. Let $X$ be a connected nonempty metric space. The span $\sigma(X)$ of $X$ is the least upper bound of the set of real numbers $r \geq 0$ satisfying the following condition: There exists a connected space $Y$ and a pair of continuous functions $f, g: Y \rightarrow X$ such that $f(Y)=g(Y)$ and $\operatorname{dist}[f(y), g(y)] \geq r$ for $y \in Y$. The surjective span $\sigma^{*}(X)$ of $X$ is defined similarly. The only difference is that we impose one additional condition on the mappings $f$ and $g$, namely, $f(Y)=X=g(Y)$. To obtain the definition of the semispan $\sigma_{0}(X)$ of $X$ we replace the condition $f(Y)=g(Y)$ in the definition of $\sigma(X)$ by the inclusion $f(Y) \subset g(Y)$, and to define the surjective semispan $\sigma_{0}^{*}(X)$ of $X$ we additionally require that $g(Y)=X$ (while $f(Y) \subset g(Y)$ ). The following inequalities were established in [2]:

$$
\begin{aligned}
& 0 \leq \sigma^{*}(X) \leq \sigma(X) \leq \sigma_{0}(X) \leq \operatorname{diam} X, \\
& 0 \leq \sigma^{*}(X) \leq \sigma_{0}^{*}(X) \leq \sigma_{0}(X) \leq \operatorname{diam} X
\end{aligned}
$$

for every connected nonempty metric space $X$.

We now introduce the notion of a directional diameter. Let $X$ be a simple closed curve in the Cartesian plane. Let $L_{\alpha}$ denote the line passing through

Received by the editors February 20, 1988.

1980 Mathematics Subject Classification (1985 Revision). Primary 54F20.

The results in this papir were announced in the Abstracts of the AMS, October 1988, Issue 59, Volume 9, Number 5, p. 425. 
the origin such that the angle between the positive $x$-axis and $L_{\alpha}$ measured counterclockwise is $\alpha, \alpha \in[0, \pi)$. We define the directional diameter $d_{\alpha}(X)$ of $X$ in the direction $\alpha$ as the length of the longest line segment (segments) with endpoints on $X$ that is parallel to $L_{\alpha}$.

Lemma 1. Let $X$ be a simple closed curve and let $d(X)=\inf \left[d_{\alpha}(X): \alpha \in\right.$ $[0, \pi)]$. Then $\sigma_{0}(X) \leq d(X)$.

Proof. Let $Y$ be a connected space and let $f, g: Y \rightarrow X$ be continuous functions such that $f(Y) \subset g(Y)$. Let $\alpha \in[0, \pi)$ be fixed but arbitrary. Let us consider a Cartesian coordinate system in which $L_{\alpha}$ is the $y$-axis. We define two sets $A$ and $B$ as follows:

$$
\begin{aligned}
& A=[y \in Y: \operatorname{Re} f(y)<\operatorname{Re} g(y)], \\
& B=[y \in Y: \operatorname{Re} f(y)>\operatorname{Re} g(y)] .
\end{aligned}
$$

The sets $A$ and $B$ are open in $Y$, and $A \cap B=\varnothing$. We shall show that $Y \neq A \cup B$. Suppose $Y=A \cup B$. Notice that then both $A$ and $B$ are nonempty. Indeed, $A=\varnothing$ implies that $\operatorname{Re} f(y)>\operatorname{Re} g(y)$ for all $y \in Y$. Let $y_{1} \in Y$ be such that $\operatorname{Re} g\left(y_{1}\right) \geq \operatorname{Re} g(y)$ for all $y \in Y$. (It suffices to consider $Y$ that is a closed subset of $X \times X$, [1, p. 209].) If $\operatorname{Re} f\left(y_{1}\right)>\operatorname{Re} g\left(y_{1}\right)$, then $f\left(y_{1}\right) \notin g(Y)$. This contradicts the assumption that $f(Y) \subset g(Y)$. Hence $A \neq \varnothing$. Similarly we argue that $B \neq \varnothing$. Hence the connectedness of $Y$ implies that there exists $y_{0} \in Y$ such that $\operatorname{Re} f\left(y_{0}\right)=\operatorname{Re} g\left(y_{0}\right)$. Therefore $\operatorname{dist}\left[f\left(y_{0}\right), g\left(y_{0}\right)\right] \leq d_{\alpha}(X)$, and consequently $\sigma_{0}(X) \leq d_{\alpha}(X)$. Finally, since $\alpha$ was arbitrary, we have $\sigma_{0}(X) \leq d(X)$.

\section{REGULAR POLYGONS}

Let $X$ be a regular polygon with $n$ sides. Suppose $n$ is odd, $A$ is a vertex of $X$, and $B C$ is the side of $X$ opposite to $A$; i.e., $\operatorname{dist}[B, A]=\operatorname{dist}[C, A]$. Let $A^{\prime}$ be the orthogonal projection of $A$ onto $B C$. Notice that $d(X)=$ $\operatorname{dist}\left[A, A^{\prime}\right]$. Suppose now that $n$ is even, the length of a side of $X$ is $\lambda$, and the radius of the circumscribed circle is $\rho$. Then $d(X)=\sqrt{4 \rho^{2}-\lambda^{2}}$.

For positive integers $m, n$, we assume the convention

$$
m(\bmod n)= \begin{cases}m, & \text { if } m \leq n \\ m-k n, & \text { if } m \in(k n,(k+1) n], k=1,2, \ldots\end{cases}
$$

Theorem 2. If $X$ is an $n$-sided regular polygon, then

$$
\sigma^{*}(X)=\sigma(X)=\sigma_{0}^{*}(X)=\sigma_{0}(X)=d(X) .
$$

Proof. We will first deal with the case when $n$ is odd. Let $A_{1}$ be a vertex of $X$. We number the remaining vertices of $X$ in a clockwise direction, $A_{2}, A_{3}, \ldots, A_{n}$. For $i=1,2, \ldots, n, A_{i} A_{j(i)}$ will denote the side of $X$ with the endpoints $A_{i}$ and $A_{j(i)}$, where $j(i)=[i+1](\bmod n)$. Let $i$ be an arbitrary 
but fixed even number from the interval $[0,2 n-2]$, and let $k_{i}=\left[\frac{i+2}{2}+\frac{n-1}{2}\right]$ $(\bmod n)$. We define functions $f, g:\left[\frac{i}{2 n}, \frac{i+2}{2 n}\right] \rightarrow X$ as follows:

(i) for $t \in\left[\frac{i}{2 n}, \frac{i+1}{2 n}\right], f(t)=A_{(i+2) / 2}$, while $g(t)$ is an affine mapping of $\left[\frac{i}{2 n}, \frac{i+1}{2 n}\right]$ onto $A_{k_{i}} A_{j\left(k_{i}\right)}$ such that $g\left(\frac{i}{2 n}\right)=A_{k_{i}}$ and $g\left(\frac{i+1}{2 n}\right)=A_{j\left(k_{i}\right)}$;

(ii) for $t \in\left[\frac{i+1}{2 n}, \frac{i+2}{2 n}\right], g(t)=A_{j\left(k_{i}\right)}$, while $f(t)$ is an affine mapping of $\left[\frac{i+1}{2 n}, \frac{i+2}{2 n}\right]$ onto $A_{(i+2) / 2} A_{j(i+2 / 2)}$ such that $f\left(\frac{i+1}{2 n}\right)=A_{(i+2) / 2}$ and $f\left(\frac{i+2}{2 n}\right)=A_{j(i+2 / 2)}$.

Notice that, according to the above formula, when $t \in\left[0, \frac{1}{2 n}\right], f(t)=A_{1}$, while $g(t)$ is an affine mapping of $\left[0, \frac{1}{2 n}\right]$ onto $A_{(n+1) / 2} A_{(n+1) / 2+1}$ such that $g(0)=A_{(n+1) / 2}, g\left(\frac{1}{2 n}\right)=A_{(n+1) / 2+1}$, and that when $t \in\left[\frac{1}{2 n}, \frac{2}{2 n}\right], f(t)$ is an affine mapping of $\left[\frac{1}{2 n}, \frac{2}{2 n}\right]$ onto $A_{1} A_{2}$, such that $f\left(\frac{1}{2 n}\right)=A_{1}, f\left(\frac{2}{2 n}\right)=A_{2}$, while $g(t)=A_{(n+1) / 2+1}$. Also, as $i$ varies through the set of even numbers $0,2, \ldots, 2 n-2$ we obtain functions $f, g:[0,1] \rightarrow X$ that are both onto and continuous, with $\operatorname{dist}[f(t), g(t)] \geq d(X)$. Hence $\sigma^{*}(X) \geq d(X)$. Since, according to Lemma $1, \sigma_{0}(X) \leq d(X)$, inequalities (1) and (2) imply that

$$
\sigma^{*}(X)=\sigma(X)=\sigma_{0}^{*}(X)=\sigma_{0}(X)=d(X) .
$$

The case when $n$ is even is dealt with analogously, with the only difference being the definition of $k_{i}$. Here $k_{i}=\left[\frac{i+2}{2}+\frac{n}{2}-1\right](\bmod n)$. Hence, while $f(0)=f(1)=A_{1}, g(0)=g(1)=A_{n / 2+1}$.

Remark. The result $\sigma^{*}(X)=\sigma(X)=\sigma_{0}^{*}(X)=\sigma_{0}(X)=\sqrt{4 \rho^{2}-\lambda^{2}}$ when $X$ is a regular polygon with an even number of sides, the length of the side is $\lambda$, and the radius of the circumscribed circle is $\rho$ was previously obtained by Thelma West [3] as a special case of an indented circle.

\section{SiMPLE CLOSED CURVES THAT ARE BOUNDARIES OF CONVEX REGIONS}

Let us first consider the case of a triangle. Let $A B C$ be a triangle with vertices $A, B$, and $C$. Let $h_{A}, h_{B}$, and $h_{C}$ be the altitudes of $A B C$ orthogonal correspondingly to $B C, A C$, and $A B$. Clearly $d(A B C)=\min \left[h_{A}, h_{B}, h_{C}\right]$. It is also easy to construct two continuous functions $f, g$ on the unit interval $I=[0,1]$ such that $f(I)=g(I)=A B C$, dist $[f(s), g(s)] \geq d(A B C)$ for $s \in I, f(0)=f(1)$, and $g(0)=g(1)$. To do this, let $H_{i}, i=1,2, \ldots, 6$, be the affine mappings such that:

$$
\begin{aligned}
& H_{1}:\left[0, \frac{1}{6}\right] \rightarrow B C \text { and } H_{1}(0)=B, H_{1}\left(\frac{1}{6}\right)=C, \\
& H_{2}:\left[\frac{1}{6}, \frac{2}{6}\right] \rightarrow A B \text { and } H_{2}\left(\frac{1}{6}\right)=A, H_{2}\left(\frac{2}{6}\right)=B,
\end{aligned}
$$


and define

$$
\begin{aligned}
& H_{3}:\left[\frac{2}{6}, \frac{3}{6}\right] \rightarrow C A \text { and } H_{3}\left(\frac{2}{6}\right)=C, H_{3}\left(\frac{3}{6}\right)=A, \\
& H_{4}:\left[\frac{3}{6}, \frac{4}{6}\right] \rightarrow B C \text { and } H_{4}\left(\frac{3}{6}\right)=B, H_{4}\left(\frac{4}{6}\right)=C, \\
& H_{5}:\left[\frac{4}{6}, \frac{5}{6}\right] \rightarrow A B \text { and } H_{5}\left(\frac{4}{6}\right)=A, H_{5}\left(\frac{5}{6}\right)=B, \\
& H_{6}:\left[\frac{5}{6}, 1\right] \rightarrow C A \text { and } H_{6}\left(\frac{5}{6}\right)=C, H_{6}(1)=A
\end{aligned}
$$

$$
\begin{aligned}
& f(s)= \begin{cases}A, & \text { if } s \in\left[0, \frac{1}{6}\right] \\
H_{2}(s), & \text { if } s \in\left[\frac{1}{6}, \frac{2}{6}\right] \\
B, & \text { if } s \in\left[\frac{2}{6}, \frac{3}{6}\right] \\
H_{4}(s), & \text { if } s \in\left[\frac{3}{6}, \frac{4}{6}\right] \\
C, & \text { if } s \in\left[\frac{4}{6}, \frac{5}{6}\right] \\
H_{6}(s), & \text { if } s \in\left[\frac{5}{6}, 1\right]\end{cases} \\
& g(s)= \begin{cases}H_{1}, & \text { if } s \in\left[0, \frac{1}{6}\right] \\
C, & \text { if } s \in\left[\frac{1}{6}, \frac{2}{6}\right] \\
H_{3}, & \text { if } s \in\left[\frac{2}{6}, \frac{3}{6}\right] \\
A, & \text { if } s \in\left[\frac{3}{6}, \frac{4}{6}\right] \\
H_{5}, & \text { if } s \in\left[\frac{4}{6}, \frac{5}{6}\right] \\
B, & \text { if } s \in\left[\frac{5}{6}, 1\right] .\end{cases}
\end{aligned}
$$

Hence the assertion of Theorem 2 holds also for all triangles.

The above is a special case of the argument used in the following:

Theorem 3. Let $D$ be a bounded convex region in the plane and let $\partial D$ be its boundary. Then

$$
\sigma^{*}(\partial D)=\sigma(\partial D)=\sigma_{0}^{*}(\partial D)=\sigma_{0}(\partial D)=d(\partial D) .
$$

Proof. Let $\varepsilon$ be a positive number such that $\varepsilon<d(\partial D)$. Inscribe a convex polygon $P$ in $\partial D$ such that $d(P) \geq d(\partial D)-\varepsilon$. Let $L_{1}, L_{2}, \ldots, L_{n}$ be the sides of $P$ numbered in their successive clockwise order. Let $B_{i}, i=$ $1, \ldots, n$, be the endpoint of $L_{i}$ that precedes $L_{i}$ in the clockwise order and let $R_{i}, i=1, \ldots, n$, be the line containing $L_{i}$. Let $A_{i}$ be a vertex of $P$ such that $\operatorname{dist}\left[R_{i}, A_{i}\right] \geq \operatorname{dist}\left[R_{i}, A\right]$ for each $A \in P$. We shall describe the motion of two points $F$ and $G$ tracing $P$. Both points will be moving only in the clockwise direction and both will return to their starting positions. Whenever $G$ travels along a side of $P, F$ will remain still at one of the vertices of $P$, and vice versa.

Let us begin with $F$ at $B_{1}$ and $G$ at $A_{1}$. 
Step 1. $G$ remains at $A_{1}$, while $F$ travels along $L_{1}$ until it reaches $B_{2}$. Notice that $A_{1}=B_{k}$ for some $k=3, \ldots, n$. Consider the lines $R_{2}, R_{k}$ containing, respectively, $L_{2}$ and $L_{k}$. There are two cases:

Case 1. $R_{2} \cap R_{k} \neq \varnothing$.

Case 2. $R_{2} \cap R_{k}=\varnothing$.

Step 2 in Case 1. Since $P$ is convex, it is contained in one of the four infinite wedges formed by $R_{2}$ and $R_{k}$. Therefore, if we consider the clockwise movement of $F$ and $G$, exactly one of them would be traveling toward the intersection point of $R_{2}$ and $R_{k}$, while the other would be traveling away from it. We choose the former to remain still while the latter travels along the side of $P$ that immediately succeeds it.

Step 2 in Case 2. We arbitrarily choose one of the points $F, G$ to remain still while the other travels along the side of $P$ that immediately succeeds it.

After we complete step $2, F$ is at $B_{i}$ and $G$ is at $B_{j}$, where $i=2$ while $j=k+1$ or $i=3$ while $j=k$. We now consider the lines $R_{i}$ and $R_{j}$ containing respectively $L_{i}$ and $L_{j}$ and repeat the procedure described in Step 2. Notice that, after finitely many steps, all of which follow the procedure described in Step 2, $F$ will return to $B_{1}$. Indeed, $F$ cannot remain indefinitely at any of the $B_{i}$ while $G$ travels along $P$. According to our algorithm, $G$ will be stopped at the vertex immediately preceding $B_{i}$ (if not earlier), and then $F$ will resume its clockwise motion. Since the same argument holds for $G$, it too will return to its initial position after finitely many steps. We also claim that whenever $F$ (or $G$ ) travels along any given side $L_{i}, G$ (or $F$ ) remains still at a vertex $B_{k}$ such that $\operatorname{dist}\left[R_{i}, B_{k}\right]=\operatorname{dist}\left[R_{i}, A_{i}\right]$.

If not, then the convexity of $P$ assures that either

$$
\operatorname{dist}\left[R_{i}, B_{k-1}\right]>\operatorname{dist}\left[R_{i}, B_{k}\right]
$$

or

$$
\operatorname{dist}\left[R_{i}, B_{k+1}\right]>\operatorname{dist}\left[R_{i}, B_{k}\right] \text {. }
$$

If (3) holds, then the position of $L_{k-1}$ with respect to $L_{i}$ is such that, according to our algorithm, $G$ had to remain at $B_{k-1}$ while $F$ traveled along $L_{i}$. Hence $F$ cannot travel along $L_{i}$ while $G$ stays at $B_{k}$. If (4) holds, then the position of $L_{k}$ with respect to $L_{i}$ is such that, according to our algorithm, $G$ had to travel along $L_{k}$ to $B_{k+1}$ while $F$ remained at $B_{i}$. Therefore in this case also $F$ cannot travel along $L_{i}$ while $G$ stays at $B_{k}$. This contradiction shows that $\operatorname{dist}\left[R_{i}, B_{k}\right]=\operatorname{dist}\left[R_{i}, A_{i}\right]$.

Let $a_{i}=\operatorname{dist}\left[A_{i}, R_{i}\right]$, and let $a=\min \left[a_{i}, i=1,2, \ldots, n\right]$. The motion of $F$ and $G$ defines two continuous functions $f$ and $g$ on a circle with $P$ as the range and such that, for every $x$ on the circle, $\operatorname{dist}[f(x), g(x)] \geq a$. Hence $\sigma^{*}(P) \geq a$. Since $a \geq d_{\alpha}(P)$ for some $\alpha$, it follows that $\sigma^{*}(P) \geq a \geq d(P)$. This, along with Lemma 1 and inequalities (1) and (2) implies that

$$
\sigma^{*}(P)=\sigma(P)=\sigma_{0}^{*}(P)=\sigma_{0}(P)=a=d(P) .
$$


We now define a continuous function $f_{1}$ on the circle in the following way:

(i) $f_{1}(x)=f(x)$ on each arc of positive Lebesgue measure on which $f$ is constant.

(ii) For each $L_{i}$, let $L_{i}^{*}$ be the arc of $\partial D$ whose endpoints coincide with the endpoints of $L_{i}$ and whose interior lies in the complement of $P$. Let $h$ be a homeomorphism from $L_{i}$ onto $L_{i}^{*}$ that fixes the endpoints of $L_{i}$. Finally, let $L_{i}^{\prime}$ be the arc on the circle that is covered by $x$ as $f(x)$ travels along $L_{i}$. For each $x \in L_{i}^{\prime}$ put $f_{1}(x)=h(f(x))$.

We define a continuous function $g_{1}$ using $g$ in the same way that we used $f$ to define $f_{1}$. Notice that $\operatorname{dist}\left[f_{1}(x), g_{1}(x)\right] \geq d(P)$ and, since $d(P) \geq d(\partial D)-\varepsilon$, we also have $\operatorname{dist}\left[f_{1}(x), g_{1}(x)\right] \geq d(\partial D)-\varepsilon$. Hence $\sigma^{*}(\partial D) \geq d(\partial D)-\varepsilon$ and, since $\varepsilon$ was arbitrary, $\sigma^{*}(\partial D) \geq d(\partial D)$. This, along with Lemma 1 and inequalities (1) and (2), implies that

$$
\sigma^{*}(\partial D)=\sigma(\partial D)=\sigma_{0}^{*}(\partial D)=\sigma_{0}(\partial D)=d(\partial D) .
$$

\section{NONCONVEX QUADRILATERALS}

We now present a nonconvex case in which all four versions of span are equal to the infimum of the set of directional diameters. We first notice that each nonconvex quadrilateral is contained in the closure of the region bounded by a triangle whose vertices are also vertices of the quadrilateral. Suppose $Q$ is a quadrilateral with vertices $A, B, C, D$ and suppose $Q$ is contained in the closure of the region bounded by the triangle $A B D$ (see Figure 1). Let $h_{D}$ be the altitude drawn from $D$ to $A B, h_{B}$ be the altitude drawn from $B$ to $A D$, and $h_{A}=\operatorname{dist}[A, D C \cup C B]$. Let $H_{i}, i=1,2, \ldots, 6$ be the affine mappings such that:

$$
\begin{aligned}
& H_{1}:\left[0, \frac{1}{6}\right] \rightarrow B A \text { and } H_{1}(0)=B, H_{1}\left(\frac{1}{6}\right)=A, \\
& H_{2}:\left[\frac{1}{6}, \frac{2}{6}\right] \rightarrow D C \cup C B \text { and } H_{2}\left(\frac{1}{6}\right)=D, H_{2}\left(\frac{2}{6}\right)=B,
\end{aligned}
$$

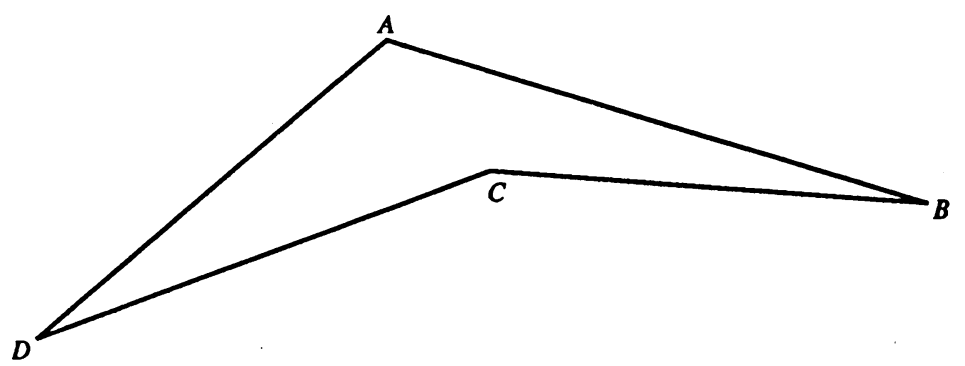

FIGURE 1 


$$
\begin{aligned}
& H_{3}:\left[\frac{2}{6}, \frac{3}{6}\right] \rightarrow A D \text { and } H_{3}\left(\frac{2}{6}\right)=A, H_{3}\left(\frac{3}{6}\right)=D, \\
& H_{4}:\left[\frac{3}{6}, \frac{4}{6}\right] \rightarrow B A \text { and } H_{4}\left(\frac{3}{6}\right)=B, H_{4}\left(\frac{4}{6}\right)=A, \\
& H_{5}:\left[\frac{4}{6}, \frac{5}{6}\right] \rightarrow D C \cup C B \text { and } H_{5}\left(\frac{4}{6}\right)=D, H_{5}\left(\frac{5}{6}\right)=B, \\
& H_{6}:\left[\frac{5}{6}, 1\right] \rightarrow A D \text { and } H_{6}\left(\frac{5}{6}\right)=A, H_{6}(1)=D .
\end{aligned}
$$

We define mappings $f, g:[0,1] \rightarrow Q$ as follows

$$
\begin{aligned}
& f(s)= \begin{cases}D, & \text { if } s \in\left[0, \frac{1}{6}\right] \\
H_{2}(s), & \text { if } s \in\left[\frac{1}{6}, \frac{2}{6}\right] \\
B, & \text { if } s \in\left[\frac{2}{6}, \frac{3}{6}\right] \\
H_{4}(s), & \text { if } s \in\left[\frac{3}{6}, \frac{4}{6}\right] \\
A, & \text { if } s \in\left[\frac{4}{6}, \frac{5}{6}\right] \\
H_{6}(s), & \text { if } s \in\left[\frac{5}{6}, 1\right],\end{cases} \\
& g(s)= \begin{cases}H_{1}(s), & \text { if } s \in\left[0, \frac{1}{6}\right] \\
A, & \text { if } s \in\left[\frac{1}{6}, \frac{2}{6}\right] \\
H_{3}(s), & \text { if } s \in\left[\frac{2}{6}, \frac{3}{6}\right] \\
D, & \text { if } s \in\left[\frac{3}{6}, \frac{4}{6}\right] \\
H_{5}(s), & \text { if } s \in\left[\frac{4}{6}, \frac{5}{6}\right] \\
B, & \text { if } s \in\left[\frac{5}{6}, 1\right] .\end{cases}
\end{aligned}
$$

Clearly, $f$ and $g$ are onto, $f(0)=f(1), g(0)=g(1)$, and $\operatorname{dist}[f(s), g(s)] \geq$ $\min \left[h_{A}, h_{B}, h_{D}\right]$. Hence

$$
\sigma^{*}(Q) \geq \min \left[h_{A}, h_{B}, h_{D}\right] .
$$

Notice also that $\min \left[h_{A}, h_{B}, h_{D}\right]$ is a directional diameter of $Q$. Therefore Lemma 1, (5), (1), and (2) imply that

$$
\sigma^{*}(Q)=\sigma(Q)=\sigma_{0}^{*}(Q)=\sigma_{0}(Q)=\min \left[h_{A}, h_{B}, h_{D}\right]=d(Q) .
$$

\section{REFERENCES}

1. A. Lelek, Disjoint mappings and the span of spaces, Fund. Math. 55 (1964), 199-214.

2. __ On the surjective span and semispan of connected metric spaces, Colloq. Math. 37 (1977), 35-45.

3. Thelma West, The span structure of some graphs and continua, Ph.D. thesis, University of Houston, 1986.

Department of Mathematical Sciences, West Chester University, West Chester, PenNSYlVANia 19383 